\title{
Optimization of Phase-Sensitive Transparent Detector for Length Measurements
}

\author{
Kyung Hoon Jun, Eerke Bunte, and Helmut Stiebig
}

\begin{abstract}
A phase selective partly transparent detector (PSTD) enables length measurement with nm-accuracy by sampling an optical standing wave. The PSTD consists of two transparent $n-i-p$ photodiodes of amorphous silicon (a-Si:H) which are embedded between three transparent conductive oxide (TCO) layers. The two photodiodes measure the intensity of an optical standing wave by means of absorption layers with thicknesses below $50 \mathrm{~nm}$ and thus, provide two photocurrents which are proportional to the intensity at their individual positions. For an optimization of the device performance, simulations based on a standard electromagnetic formalism were performed. The considered thin-film structure is a glass/TCO/n-i-p/TCO/n-i-p/TCO layer sequence. The aim was to design a layer stack which avoids significant distortions of the standing wave while the phase shift between the photocurrents approximately amounts to $90^{\circ}$, since this will minimize the measurement error. The comparison of experimentally determined and simulated data shows that a further adjustment of the fabricated PSTD into an ideal thickness scheme is necessary to enhance the device performance.
\end{abstract}

Index Terms-Amorphous semiconductors, optical distance measurement, optical interferometry, photodetector, silicon, thin films.

\section{INTRODUCTION}

$\mathbf{L}$ ENGTH measurement and position detection in the $\mathrm{nm}$ range is important in coordinate measuring systems, lithographers and position calibrators [1]. For these tasks laser interferometers are commonly used, since they offer a high measurement precision. The state-of-the-art technology is the Michelson interferometer.

We have realized an alternative, a so-called standing wave interferometer for length measurements, which utilizes an optical standing wave and has the potential to substitute the Michelson interferometer as a more inexpensive and compact alternative. The utilization of an optical standing wave for length measurement was investigated in the past [2]-[4], but our approach reduces the number of optical components to a minimum. Another interesting application of optical standing waves are Fourier spectrometers [5], [6], which allow the determination of different wavelengths by transparent detectors.

The principle of our standing wave interferometer is based on the sampling of an optical standing wave. An optical standing wave can be generated by a laser, e.g., a He-Ne laser with a

\footnotetext{
Manuscript received October 19, 2004; revised March 24, 2005. This work was supported by the "Deutsche Forschungsgemeinschaft" (Sti 181/1-2). The review of this paper was arranged by Editor K. Najafi.

The authors are with the Forschungszentrum Jülich GmbH, Institute of Photovoltaics (IPV), D-52425 Jülich, Germany (e-mail: samuel_jun@kaist.ac.kr).

Digital Object Identifier 10.1109/TED.2005.850614
}

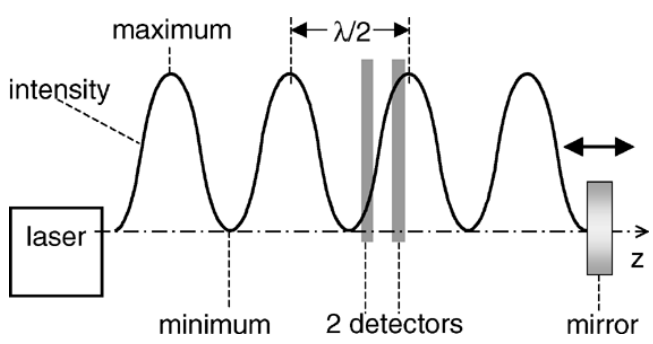

Fig. 1. Setup for the generation of an optical standing wave. Two detectors in the standing wave enable bidirectional fringe counting.

wavelength of $\lambda=633 \mathrm{~nm}$, which is orthogonally incident onto a mirror.

This setup leads to an interference of two electromagnetic waves propagating in opposite direction forming a standing wave. The standing wave is characterized by its intensity profile $I$. The intensity of an ideal optical standing wave (no light distortion by the detector) is proportional to the square of the electrical field $E$ of the wave [(1)]

$$
I(z) \propto E_{z}^{2}+E_{-z}{ }^{2}-2 E_{z} E_{-z} \cos (2 k z) \quad \text { with } k=\frac{2 \pi}{\lambda} .
$$

where $E_{z}$ and $E_{-z}$ describe the incident and the reflected waves, respectively. $I$ consists of minima and maxima which appear with a period of $\lambda / 2$ (see Fig. 1). At the mirror always occurs a minimum, since here the electric field disappears. Hence, the intensity profile is spatially fixed and can be shifted when the mirror is moved along the $\mathrm{z}$-axis. By sampling the intensity with a partly transparent detector, information about the mirror displacement can be obtained.

The task of the detector is to generate a photocurrent corresponding to the standing wave intensity at its position. Moving the mirror along the optical axis shifts the intensity profile through the detector. Consequently a mirror-position-dependent photocurrent can be generated.

However, with one detector the direction of the mirror movement can not be determined. To identify the direction, two detectors should be implemented into the standing wave providing two independent photocurrents (see Fig. 1). If the mirror is moved, two periodical photocurrents can be displayed on the $\mathrm{x}$ - and $\mathrm{y}$-channel of an oscilloscope as a Lissajous curve. Those can be evaluated for bidirectional fringe counting - a standard method for interferometrical length measurements. Then the detection of the moving direction of the mirror is possible and the measurement accuracy is enhanced. With this method, the displacement can be determined with an accuracy shorter than the period of the standing wave. 


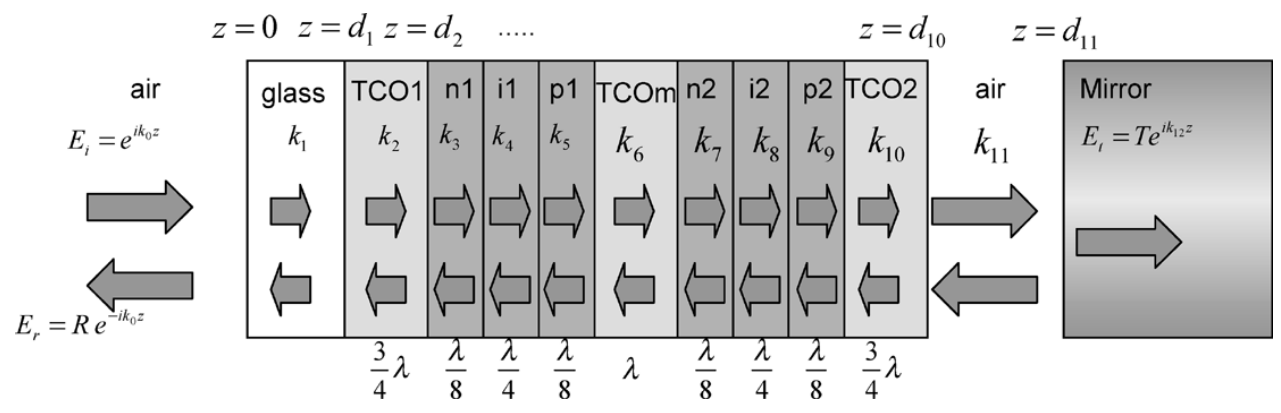

Fig. 2. Layer scheme of the simulated PSTD. Two n-i-p diodes are embedded between three transparent contact layers. An example of optimum thickness values are displayed in this figure. Note that the $\lambda$ value represents the wavelength in each material. For example, $\lambda$ in TCO is $\lambda_{\text {air }} / n_{\mathrm{TCO}}$, where $n_{\mathrm{TCO}}$ denotes the refractive index of TCO. Therefore the thicknesses of layers are; TCO1: $250 \mathrm{~nm}, \mathrm{n} 1: 22 \mathrm{~nm}, \mathrm{i} 1: 44 \mathrm{~nm}, \mathrm{p} 1: 23 \mathrm{~nm}, \mathrm{TCOm}: 333 \mathrm{~nm}, \mathrm{n} 2: 22 \mathrm{~nm}, \mathrm{i} 2: 44 \mathrm{~nm}, \mathrm{p} 2: 23$ $\mathrm{nm}$, and TCO2: $250 \mathrm{~nm}$. We assumed that the thickness of the glass is $1.117556 \mathrm{~mm}(0.48 \mathrm{inch})$ for the simulation.

For two sinusoidal photocurrents, the Lissajous figures form ellipses. The shape of the ellipse depends on the phase shift $(\gamma)$ between the photocurrents. For $\gamma=0^{\circ}, 180^{\circ}, 360^{\circ}, \ldots$ (the values will be simply referred as $0^{\circ}$ from now on), the ellipse shrinks to a line and the direction of the mirror movement can not be determined. For a phase shift of $\gamma=90^{\circ}, 270^{\circ}, 450^{\circ}, \ldots$ (the values will be simply referred as $90^{\circ}$ from now on), the Lissajous figure depicts a circle and the two signals build an orthogonal system. These cases are desirable, since they are optimal for the least measurement error [7]. A deviation from $\gamma=90^{\circ}$ leads to an increase of the measurement error. Furthermore, a deviation of the photocurrents from a sine function increases the measurement error.

We have developed an integrated device consisting of two $\mathrm{n}$-i-p photodiodes with an absorption layer thickness shorter than the wavelength of the incident light. The two diodes of the phase selective partly transparent detector (PSTD) are embedded between three transparent conductive oxide (TCO) contact layers which border the amorphous silicon $(\mathrm{a}-\mathrm{Si}: \mathrm{H})$ n-i-p photodiodes (see Fig. 2). Such devices can be used to sample the intensity profile of a standing wave and have proven to deliver two independent photocurrents enabling bidirectional fringe counting [8]. The measured photocurrents of the fabricated PSTD do not describe pure sine functions but show distortions, which lead to nonlinearities and thus to an increase of the measurement error [9].

We have performed numerical simulations to optimize the device layer structure so that the phase difference between the photocurrents of the diodes as functions of the mirror displacement is close to $90^{\circ}$ and the photocurrents show almost sinusoidal behavior. A comparison with experimental data is incorporated into the discussion of the simulations.

\section{EXPERIMENT}

For the fabrication of the devices we took benefit from the well-developed a-Si:H based thin-film process for solar cells [10]. A $10 \times 10 \mathrm{~cm}^{2}$ Corning glass, type $1737 \mathrm{~F}$, was used as substrate for the thin-film devices. The preparation of the TCO layers was performed by rf-magnetron sputtering of aluminum doped zinc oxide $(\mathrm{ZnO})$. The amorphous silicon layers were deposited in a multichamber PECVD system at $210^{\circ} \mathrm{C}$ as an $n$-i-p layer sequence.
The absorber layer, which is the i-layer, was prepared using a gas mixture of silane, methane and hydrogen. Thus, it is composed of amorphous silicon carbide $(\mathrm{a}-\mathrm{SiC}: \mathrm{H})$ and has an optical bandgap of $\mathrm{E}_{\mathrm{g}}=2.0 \mathrm{eV}$. The p-layer was deposited with a-SiC:H $\left(\mathrm{E}_{\mathrm{g}}=1.9 \mathrm{eV}\right)$, whereas the n-layer consists of a-Si:H $\left(\mathrm{E}_{\mathrm{g}}=1.75 \mathrm{eV}\right) . \mathrm{p}$ - and n-type doping were performed by adding trimethylboron and phosphine to the process gases, respectively. The deposition procedure has a reproducibility of approximately $\pm 20 \mathrm{~nm}$ for the TCO layer thickness and approximately $\pm 5-10 \mathrm{~nm}$ for the thickness of the amorphous layers.

The phase shift between the photocurrents is determined by the design of the layer stack, e.g., the layer thicknesses, refractive indices, etc. The phase shift can not be adjusted after the deposition of the thin-film layers is completed. Therefore, each layer has to be prepared carefully, to achieve the aimed phase shift of $90^{\circ}$.

Finally, the detectors were patterned using photolithography and reactive ion etching. The first-deposited diode 1 has an area of $10.7 \mathrm{~mm}^{2}$, while diode 2 is slightly smaller $\left(8.9 \mathrm{~mm}^{2}\right)$ to enable a contacting of the underlying diode 1 . The photocurrent measurements within the standing wave have been performed using a stabilized He-Ne laser $\left(\lambda_{\text {air }}=633 \mathrm{~nm}\right)$ with a power of $0.92 \mathrm{~mW}$.

\section{MODEL}

Due to the relatively high absorption coefficients of the layers, a conceptual prediction of the standing waves within the device is difficult. A detailed electromagnetic simulation of the device is performed in order to develop further optimization criteria and to achieve a better understanding of the wave propagation through the multilayer stack.

Fig. 2 shows the layer scheme of the PSTD. To simulate this structure, we assumed a plane wave which normally impinges (TEM mode) on the glass side. In Fig. $2, k_{j}$ is the wave number in the $j$ th region. The incident electric field has the unit amplitude. $R$ is the reflectance and $T$ is the transmittance. We considered silver as a nonideal mirror. The electric field ( $x$ component) in the $j$ th region is

$E_{j}=E_{j}\left(d_{j-1}\right) \exp \left[i k_{j}\left(z-d_{j-1}\right)\right]+E_{j}\left(d_{j}\right) \exp \left[-i k_{j}\left(z-d_{j}\right)\right]$. 
The coefficients $E_{j}\left(d_{j-1}\right)$ and $E_{j}\left(d_{j}\right)$ represent the amplitudes of the right-going and the left-going fields in the $j$ th region, respectively. We calculated the absorbance [11] of the i-layer, which may in diode 1 be represented by

$$
A_{\text {diode } 1}=\operatorname{Re}\left\{\frac{E_{4}\left(d_{4}\right) H_{4}^{*}\left(d_{4}\right)-E_{4}\left(d_{3}\right) H_{4}^{*}\left(d_{3}\right)}{E_{i}(0) H_{i}^{*}(0)}\right\} .
$$

The direction of the $E$ and $H$ fields was chosen to match the propagation of the TEM wave. The absorbance is normalized by the incident wave. The absorbance of diode 2 may be expressed in a similar way. The magnetic field is obtained from the Maxwell's curl equation $\nabla \times \mathbf{E}=i \varpi \mu_{0} \mathbf{H}$, assuming that the media are nonmagnetic. The coefficients $E_{j}\left(d_{j-1}\right)$ and $E_{j}\left(d_{j}\right)$ are determined by means of a matrix formalism [12]. This approach correlates the input and the output electric field vectors of the whole layer stack by a linear function and enables the determination of the coefficients at each interface in an iterative way.

Assuming that the photocurrent in each diode can only be attributed to the absorbed light in the i-layer, the absorbance of the i-layer is proportional to the photocurrent. Thus, absorbance and photocurrent can be used synonymously. In the following, the wave form within the device will be discussed by the absorbance of the diodes.

We deduced the dielectric functions of the layers by measuring the transmittance and the reflectance of individual thin films deposited on the glass substrate. We also determined the growth rate of each layer by this method.

We calculated the mirror-position-dependent photocurrent. Initially the mirror was assumed to be placed $10 \mathrm{~cm}$ behind the device $\left(d_{10}+10 \mathrm{~cm}=d_{11}\right)$. The simulations were carried out for an increased air gap up to $d_{10}+10 \mathrm{~cm}+633 \mathrm{~nm}=d_{11}$. The displayed phase shift value was obtained by fitting the curves by ideal sine functions and determining the phase difference between the two sine functions. The aim was to find layer thicknesses for the given layer sequence, which yield photocurrents with low distortions and a phase shift near $90^{\circ}$.

\section{Simulation Results}

One prerequisite to receive distortion-free photocurrents is the minimization of internal reflections. A way to achieve this is to ensure high transparency of the device at $\lambda_{\text {air }}$ of the laser light. Thus, a layer stack, which shows highest possible transmission, has to be defined.

As a first approach to define a layer stack that ensures photocurrents with low distortions and $90^{\circ}$ phase shift, we designed the layer thicknesses neglecting internal reflections and absorption losses in any layer. Fig. 2 contains possible layer thicknesses for maximum transparency. The total thickness of the device is a multiple of $\lambda$ and the TCO1 and TCO2 act as anti reflection coatings. This guarantees high transmission. For a layer system with no absorption and no internal reflections, the phase shift between the photocurrents is determined by the distance between the centers of the two i-layers, which is given by the sum of i1/2, p1, TCOm, n2, and i2/2. However, the thickness values as given in Fig. 2 result in a phase shift of $540^{\circ}$, which corresponds to a multiple of $\lambda / 2$. Thus, the shape of the

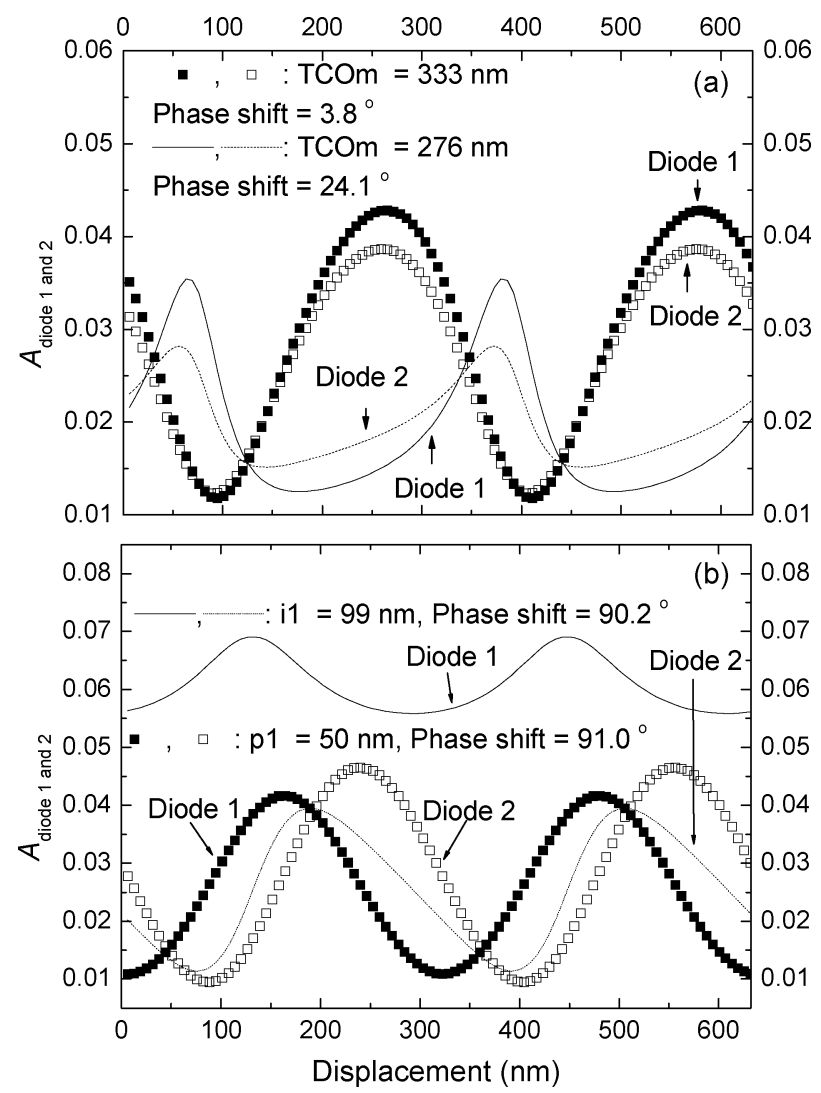

Fig. 3. (a) Absorbances of diode 1 and 2 at different TCOm thicknesses and (b) at increased i1 layer thickness and p1 layer thickness. The other thicknesses are the same as in Fig. 2. The phase shift is defined as the phase difference of the fitted sine waves of the absorbances (photocurrents).

Lissajous curve shrinks to a single line (will be discussed with Fig. 4) and the direction of the mirror movement can not be determined and the advantage of enhanced accuracy is lost. Thus, a compromise between a maximum transmission and a phase shift close to $90^{\circ}$ needs to be found.

In the following numerical simulations, the dielectric functions $n$ and $\kappa$ have been taken into account for each individual layer to study the influence of the real and imaginary part of the dielectric function on the wave propagation within the thin-film layer stack. Fig. 3(a) and (b) show the simulated absorbances $A_{\text {diode } 1}$ and $A_{\text {diode } 2}$ and Fig. 4 shows the corresponding Lissajous curves. In Fig. 3(a) and (b), the differences of the direct current (dc) levels and the amplitudes between the two diodes are attributed to the slightly higher light absorption in the diode 1. A mirror displacement of $633 \mathrm{~nm}$ results in a photocurrent (represented by the absorbance) that displays two intensity periods. In Fig. 4, for clear comparison of the shapes, we removed the dc parts and normalized the alternating current (ac) signals to vary within -0.5 and 0.5 .

The simulation of the layer structure in Fig. 2 is shown in Fig. 3(a) ( $\square$ and $\square$ ) and Fig. 4 (- $\square$-). The phase shift of the two photocurrents results in $3.8^{\circ}$. Thus, the corresponding Lissajous curve almost shrinks to a single line. In fact, a phase of $0^{\circ}$ can not be observed, since the absorption in the layers was taken into account and has an influence on the phase shift.

To obtain the desired phase shift of $90^{\circ}$ between the photocurrents, the thicknesses of the individual layers have to be 


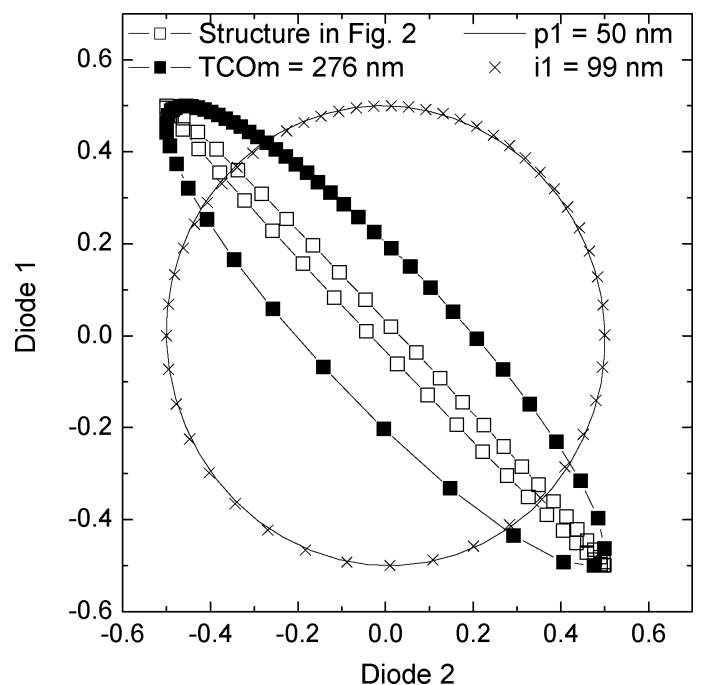

Fig. 4. Corresponding Lissajous figures to the photocurrents shown in Fig. 3.

changed. In the following we discuss three cases: 1) a change of the TCOm layer; 2) a change of the p-layer; and 3) a change of the i-layer, while the other layers were kept constant as denoted in Fig. 2. That means, the proposed variations of the layer thicknesses result in a deviation from the total optical layer thickness of a multiple of $\lambda$ for an ideal device.

Let us first focus on changing the TCOm layer. From the simple point of view - neglecting absorption and internal reflections - a phase shift of $90^{\circ}$ might be reached for a TCOm layer of $\lambda_{\mathrm{TCO}}-\lambda_{\mathrm{TCO}} / 8=7 \lambda_{\mathrm{TCO}} / 8$ thickness. However, the simulations considering absorption and internal reflections have revealed, that the phase shift of $90^{\circ}$ cannot be implemented by any change of the TCOm layer thickness. Fig. 3(a) (- and ----) shows the simulated photocurrents for a TCOm layer of $276 \mathrm{~nm}$. Fig. 4 (- $\square$-) depicts the corresponding Lissajous curves. The phase shift between the photocurrents amounts to $24.1^{\circ}$. This is the highest $\gamma$ that can be reached by varying the TCOm layer thickness. Also the wave forms are significantly distorted in this case. Due to the absorption in the TCO layers and internal reflections at the TCO/a-Si:H interfaces, a variation of the TCO layers affects the wave propagation. Thus, a change of the TCOm layer is no mean to adjust a phase of $90^{\circ}$.

Next, we have modified the p1 layer to $\mathrm{p} 1=0.27 \lambda_{\mathrm{a}-\mathrm{SiC}: \mathrm{H}}$ $(50 \mathrm{~nm})$ keeping the other parameters as in Fig. 2. Fig. 3(b) ( and $\square$ ) shows the simulated absorbance. The corresponding Lissajous curves are given in Fig. 4 (-). As we can see, increasing the $\mathrm{p} 1$ layer enables our goal of sine shaped photocurrents and a phase shift of the currents close to $90^{\circ}$. Note that instead of varying the $\mathrm{p} 1(\mathrm{a}-\mathrm{SiC}: \mathrm{H})$ layer also a variation of the $\mathrm{n}$-layer (a-Si:H) can lead to a phase shift near $90^{\circ}$. However, the former solution is more preferable due to the lower absorption losses within the carbon alloyed layer. This results in a higher transmission and the difference of the waves $E_{z}$ and $E_{-z}$ is smaller (see (1)). Therefore, the intensity $I$ in both diodes and thus, the two photocurrents have almost the same values.

Finally, we have varied the i1-layer to i1 $=0.57 \lambda_{\mathrm{a}-\mathrm{Si}: \mathrm{H}}(99$ $\mathrm{nm})$ keeping the other parameters as in Fig. 2. This leads to a phase shift of $90.2^{\circ}$, however the absorbance profile is signifi-

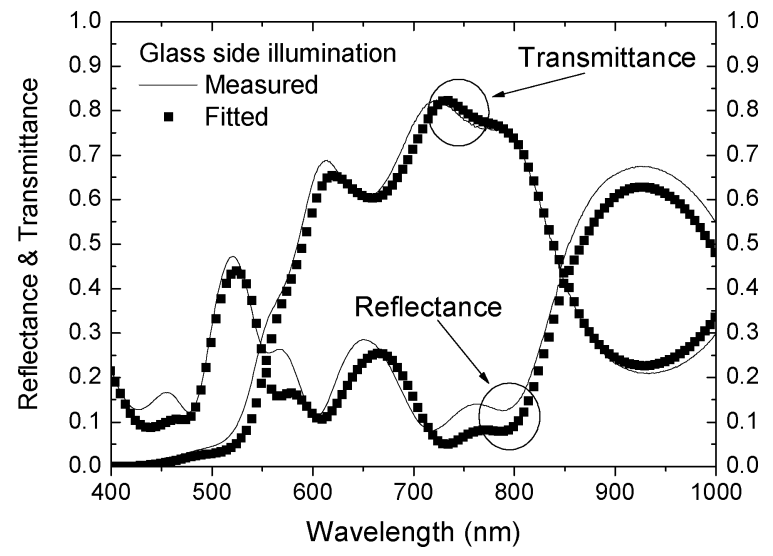

Fig. 5. Measured and simulated reflectance and transmittance behavior of a fabricated PSTD. The thicknesses of the layers are: TCO1: $265 \mathrm{~nm}, \mathrm{n} 1: 20 \mathrm{~nm}$, i1: $43 \mathrm{~nm}, \mathrm{p} 1: 42 \mathrm{~nm}$, TCOm: $319 \mathrm{~nm}, \mathrm{n} 2: 22 \mathrm{~nm}, \mathrm{i} 2: 44 \mathrm{~nm}, \mathrm{p} 2: 23 \mathrm{~nm}$, and TCO2: $244 \mathrm{~nm}$.

cantly distorted as is shown in Fig. 3(b) (- and ----). Further disadvantages are the increase of the dc part and the reduction of the ac part of the photocurrents [13].

The simulations have shown that a phase shift of $90^{\circ}$ and nearly undistorted sinusoidal photocurrents can be reached by an increased p1-layer.

\section{EXPERIMENTAL RESULTS AND DISCUSSION}

As proposed by the above-presented simulation results we have prepared PSTD with a thickness scheme according to Fig. 2, but with an enlarged p1-layer by around $20 \mathrm{~nm}$, to reach the aimed phase difference near $90^{\circ}$ while the photocurrents are not significantly distorted.

Fig. 5 shows the measured reflection and transmission curves of the fabricated thin-film device. At 610-nm wavelength, the interference pattern exhibits a maximum in transmission $(\sim 69 \%)$ and a minimum in reflection $(\sim 11 \%)$, respectively. At the laser wavelength of $633 \mathrm{~nm}$, the transmission amounts to $\sim 64 \%$ and the reflection to $\sim 24 \%$, respectively. In Fig. 5, additionally the simulated data of reflection and transmission are plotted. A good agreement between simulated and measured spectra is achieved using layer thicknesses similar to the values estimated from the deposition rates of individual thin layers. The simulated layer stack (Structure 1) has the thickness values of the layers in the fabricated PSTD system (TCO1: $265 \mathrm{~nm}, \mathrm{n} 1: 20 \mathrm{~nm}, \mathrm{i} 1: 43 \mathrm{~nm}$, p1: $42 \mathrm{~nm}$, TCOm: $319 \mathrm{~nm}, \mathrm{n} 2: 22 \mathrm{~nm}, \mathrm{i} 2: 44 \mathrm{~nm}, \mathrm{p} 2: 23 \mathrm{~nm}$, TCO2: $244 \mathrm{~nm}$ ), which were deduced by fitting the calculated transmittance and reflectance to the measured curves using a commercial software tool [14]. The thickness of the individual layers estimated by the deposition rate and the data used for the simulations differ less than $15 \mathrm{~nm}$, which is within the uncertainty limit of the deposition parameters.

Fig. 6(a) shows the normalized absorbances (or the photocurrents) of a deposited PSTD and the simulation of a structure based on the layer thicknesses derived from the former fitting. Fig. 6(b) shows the corresponding Lissajous curves. It demonstrates that the simulation can approximately describe the real device. The phase of simulated Structure 1 amounts to $109.7^{\circ}$, while the fabricated PSTD exhibits about $129^{\circ}$ phase shift. 

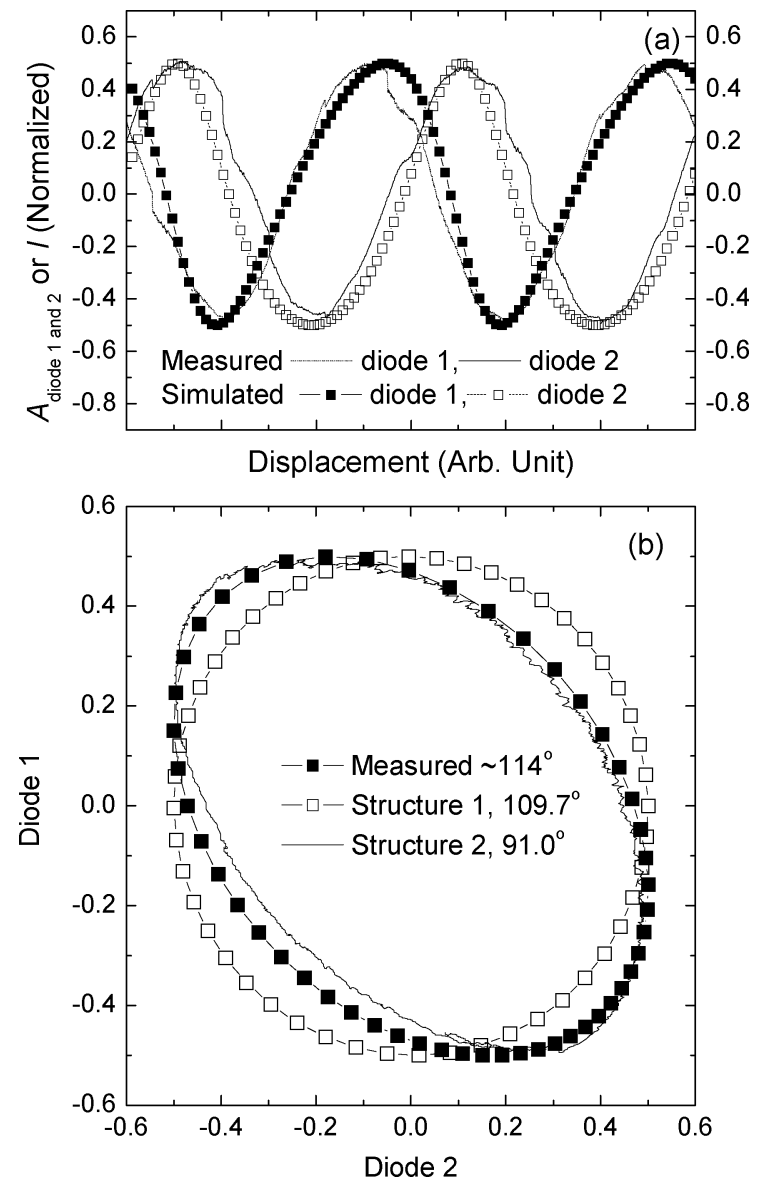

Fig. 6. (a) Normalized photocurrents of a fabricated PSTD and normalized absorbances of its simulation (Structure 1). The thicknesses of the layers are: TCO1: $265 \mathrm{~nm}, \mathrm{n} 1: 20 \mathrm{~nm}$, i1: $43 \mathrm{~nm}, \mathrm{p} 1: 42 \mathrm{~nm}$, TCOm: $319 \mathrm{~nm}, \mathrm{n} 2: 22 \mathrm{~nm}$, i2: $44 \mathrm{~nm}, \mathrm{p} 2: 23 \mathrm{~nm}$, and TCO2: $244 \mathrm{~nm}$. (b) Corresponding Lissajous curves. A simulation at an ideal thickness scheme with an increase p1 into $50 \mathrm{~nm}$ shown in Figs. 3(b) and 4 is redisplayed as Structure 2.

The reality may differ from the simulation due to some facts. Firstly, a certain amount of the thickness uncertainty in the substrate and the thin-film layers may cause differences. Secondly, the employed dielectric function for the simulation can differ from the values in the detector system due to the fact that the underlying layers can affect the growth. The underlying layers are possible to alter the dielectric function which might be different from films deposited on glass substrates. Thirdly, we assumed the ideal conversion of the intensity profile in the i-layers into the photocurrents and neglected parasitic effects such as the collection of photo-generated carriers in the doped layer and recombination losses of carriers in the i-layer.

Structure 2 corresponds to the ideal thickness scheme as given in Fig. 2 - but with an increased p1-layer (TCO1: $250 \mathrm{~nm}, \mathrm{n} 1: 22$ nm, i1: 44 nm, p1: $50 \mathrm{~nm}$, TCOm: 333 nm, n2: $22 \mathrm{~nm}, \mathrm{i} 2: 44 \mathrm{~nm}$, p2: $23 \mathrm{~nm}$, TCO2: $250 \mathrm{~nm}$ ) (see also Figs. 3(b) and 4). The simulation demonstrates, that a phase shift near $90^{\circ}$ and photocurrents with low distortions are possible for a PSTD. The comparison of Structure 1 and Structure 2 indicates that small changes in layer thicknesses have rather high impact on the device performance, namely the phase shift. Thus, for an optimization of the fabricated PSTD the deposited layers should exhibit better thickness accuracy. However, the accuracy of the layer thicknesses is limited by the uncertainties of the production process.

For this new type of position detector the displacement of the mirror can be determined with an accuracy of up to $\pm 15 \mathrm{~nm}$ [13] using the Heydemann algorithm [15]. This is a factor of $10-100$ higher than the values reported for state-of-the-art Michelson interferometers. Since the standing wave detector is based on an integrated detector concept, the alignment of the detector within a standing wave is easy. The sensor can be used for applications were limited resolution is necessary and a low cost approach is required. The main challenge for further improvement lies in the optical adjustment of the layer system to achieve two undisturbed sinusoidal shaped photocurrents with a phase shift of $90^{\circ}$. For this task, optical simulations can be a useful tool.

\section{SUMMARY}

We have investigated the PSTD system by an electromagnetic simulation of the multilayer system. We have achieved similar Lissajous curves for the fabricated and the simulated PSTD. Thus, the simulation can predict the tendency of the photocurrents within a reliable range.

Furthermore, the results show that a thicker p1-layer is a possibility to achieve photocurrents with low distortion and a phase shift near 90. This leads to Lissajous figures close to a perfect circle, which is a prerequisite for highest measurement accuracy.

Finally, the comparison of simulation and experiment reveals that a further adjustment of the deposited PSTD can lead to a higher device performance.

\section{ACKNOWLEDGMENT}

The authors thank F. Finger and A. Lambertz for the support of a-Si:H deposition, H. Siekmann and G. Schöpe for sputtering of TCO layers, and H.-J. Büchner and V. Mandryka for discussion and measurements.

\section{REFERENCES}

[1] N. Bobroff, "Recent advances in displacement measuring interferometry," Meas. Sci. Technol., vol. 4, pp. 907-926, 1993.

[2] H. Büchner, "Stehende-Wellen-Interferometer zur Messung von Optischen Gangunterschieden,” Deutsches Patent DE 3300369, 1983.

[3] M. Sasaki, X. Mi, and K. Hane, "Standing wave detection and interferometer application using a photodiode thinner than optical wavelength," Appl. Phys. Lett., vol. 75, no. 14, pp. 2008-2010, 1999.

[4] Y. Li, X. Mi, M. Sasaki, and K. Hane, "Precision optical displacement sensor based on ultrathin film photodiode type optical interferometers," Meas. Sci. Technol., vol. 14, pp. 479-483, 2003.

[5] H. L. Kung, D. A. B. Miller, P. Atanackovic, C. C. Lin, J. S. Harris Jr., L. Carraresi, J. E. Cunningham, and W. Y. Jan, "Wavelength monitor based on two single-quantum-well absorbers sampling a standing wave pattern," Appl. Phys. Lett., vol. 76, no. 22, pp. 3185-3187, 2000.

[6] H. L. Kung, S. R. Bhalotra, J. D. Mansell, D. A. B. Miller, and J. S. Harris Jr., "Standing-wave transform spectrometer based on integrated MEMS mirror and thin-film photodetector," IEEE J. Select. Topics Quantum Electron., vol. 8, no. 1, pp. 98-105, Jan.s 2002.

[7] U. Gerhardt, "Signalverarbeitung in der Interferenzoptischen Meß- und Sensortechnik," Ph.D. dissertation, Univ. Ilmenau, Ilmenau, Germany, 2002.

[8] H. Stiebig, H.-J. Büchner, E. Bunte, V. Mandryka, D. Knipp, and G. Jäger, "Standing-wave interferometer," Appl. Phys. Lett., vol. 83, pp. 12-14, 2003. 
[9] H. J. Büchner, E. Bunte, V. Mandryka, H. Stiebig, and G. Jaeger, "Standing-wave interferometer based on partially transparent photodiodes," in Proc. SPIE Int. Soc. Opt. Eng., vol. 5144, 2003, pp. 218-226.

[10] B. Rech, O. Kluth, T. Repmann, T. Roschek, J. Springer, J. Müller, F. Finger, H. Stiebig, and H. Wagner, "New materials and deposition techniques for highly efficient silicon thin film solar cells," Sol. Energy Mater. Sol. Cells, vol. 74, pp. 439-447, 2002.

[11] F. Leblanc, J. Perrin, and J. Schmitt, "Numerical modeling of the optical properties of hydrogenated amorphous-silicon-based p-i-n solar cells deposited on rough transparent conducting oxide substrates," J. Appl. Phys., vol. 75, pp. 1074-1087, 1994.

[12] Z. Knittl, Optics of Thin Films. New York: Wiley, 1976.

[13] E. Bunte, V. Mandryka, K. H. Jun, H.-J. Büchner, G. Jäger, and H. Stiebig, "Thin transparent n-i-p-photodiodes for length measurements," Sensors and Actuat. A, vol. 113, pp. 334-337, 2004.

[14] VASE, Version 2.46, Wollam, Lincoln, NE, 1995.

[15] P. L. M. Heydemann, Appl. Opt., vol. 20, pp. 3382-3384, 1981.

Kyung Hoon Jun received the M.S. and Ph.D. degrees from the Electrical Engineering Department, Korea Advanced Institute of Science and Technology (KAIST), Daejon, Korea, in 1995 and 2000, respectively. His Ph.D. research dealt with the amorphous silicon multilayer and its solar cells.

He was a Research Professor at KAIST from 2001 to 2003, and a Researcher with the Institute of Photovoltaics at Research Centre Jülich, Jülich, Germany, during 2000-2001 and 2003-2004.
Eerke Bunte received the diploma and Ph.D. degree in electrical engineering from the Technical University of Braunschweig, Braunschweig, Germany, in 2000 and 2004, respectively. His Ph.D. research dealt with a photodetector for length measurements.

He has been with the Institute of Photovoltaics at Research Centre Jülich, Jülich, Germany, since 1999. His research interests include thin-film silicon solar cells and optical sensors for different applications.

Helmut Stiebig received the Ph.D degree in electrical engineering from the Aachen University of Technology, Aachen, Germany, 1997.

Since 1992, he has been with the Institute of Photovoltaics. Research Center Jülich, Jülich, Germany. He is currently head of the research group "Device Analysis and Sensor Technology". His research interests include the investigation of the optoelectronic properties of amorphous and microcrystalline silicon and related materials, development of innovative device structures for solar cell applications, device analysis and numerical modeling, and the development of optical sensors based on thin-film technology. 